\title{
Capsule Commentary on Phillips et al., Year-End Clinic Handoffs: A National Survey of Academic Internal Medicine Programs Running Title: National Survey of Year-End Clinic Handoffs
}

\author{
Hirofumi Yoshida, MD \\ Department of General Internal Medicine, Rakuwakai Otowa Hospital, Kyoto, Japan.
}

J Gen Intern Med 32(6):684

DOI: $10.1007 / \mathrm{s} 11606-017-4020-3$

(c) Society of General Internal Medicine 2017

$\mathrm{C}$ onsiderable attention has been paid to the quality of inpatient handoffs. Less attention has been paid to endof-residency continuity clinic handoffs. Previous studies have suggested that high-risk resident patients have high no-show rates for the initial continuity visit and frequently have delayed follow-up and treatment ${ }^{1}$ and that improving ambulatory handoffs reduces acute care clinic visits. ${ }^{2}$ As part of an annual Association of Program Directors in Internal Medicine (APDIM) 2014 survey, Phillips and colleagues assessed year-end internal medicine residency clinic handoffs. ${ }^{3}$ They found that most programs lack formal continuity clinic handoff processes, educational activities or systems for evaluating these handoffs.

The authors suggest that programs should: (1) identify highrisk patients, possibly for transfer to an attending provider, and (2) implement systematic, face-to-face handoffs from the sender to the receiver to optimize the exchange. One benefit from these suggestions is that it makes incoming residents more responsible for their assigned patients. Previous studies have found that residents often fail to take ownership of their continuity patients until they have seen them in the clinic. ${ }^{1}$

While ambulatory patients are somewhat protected by the fact that resident panels are invariably part of an attending's practice, improvement in handoffs is a reasonable priority. One problem is the lack of overlap between graduating residents and incoming interns. It is possible that a simple email reminder message could increase the quality of handoffs without requiring overlap or protected time to achieve them. ${ }^{4}$ Another possibility would be to standardize a handoff template, much like as has been done in the hospital setting, to make sure that important elements of the handoff occur. In addition, a simple intervention involving letters to the patients from outgoing and incoming residents resulted in improved follow-up rates. ${ }^{5}$ Given that most programs have formal education on inpatient handoffs, incorporating ambulatory handoffs into this seems a natural extension. Future research should focus on the important elements for ambulatory handoffs, assessing the impact of poor handoffs on patient outcomes in the ambulatory setting and whether or not interventions such as those suggested by the author improves outcomes.

Corresponding Author: Hirofumi Yoshida, MD; Department of General Internal MedicineRakuwakai Otowa Hospital, Otowachinjicho 2, Yamashina-ku, Kyoto, Japan (e-mail: hirofumi.1623. yoshida@gmail.com).

\section{Compliance with Ethical Standards:}

Conflict of Interest: The author has no conflicts of interest with this article.

\section{REFERENCES}

1. Pincavage AT, Ratner S, Prochaska ML, Prochaska M, Oyler J, Davis AM, et al. Outcomes for resident-identified high-risk patients and resident perspectives of year-End continuity clinic handoffs. J Gen Intern Med. 2012;27(11):1438-44.

2. Pincavage AT, Prochaska M, Dahlstrom M, Lee WW, Beiting KJ, Ratner S, et al. Patient safety outcomes after two years of an enhanced internal medicine residency clinic handoff. Am J Med. 2014;127(1):96-9.

3. Phillips E, Harris C, Lee WW, Pincavage AT, Ouchida K, Miller RK, Chaudhry S, Arora VM. Year-end clinic handoffs: a national survey of academic internal medicine programs. J Gen Intern Med. 2017. doi:10. 1007/s11606-017-4005-2.

4. Donnelly MJ, Clauser JM, Weissman NJ. An intervention to improve ambulatory care handoffs at the end of residency. J Grad Med Educ. 2012;4(3):381-4.

5. Pincavage AT, Lee Ww, Venable LR, Prochaska M, Staisiunas DD, Beiting KJ, et al. "Ms. B changes doctors": using a comic and patient transition packet to engineer patient-oriented clinic handoffs (EPOCH). J Gen Intern Med. 2015;30(2):257-60. 